\title{
Genital tract group B streptococcal colonization in pregnant women: a South Indian perspective
}

\author{
Vijayan Sharmila ${ }^{1}$, Noyal Mariya Joseph ${ }^{2}$, Thirunavukkarasu Arun Babu ${ }^{3}$, Latha Chaturvedula ${ }^{4}$, \\ Sujatha Sistla ${ }^{4}$
}

${ }^{1}$ Department of Obstetrics and Gynaecology, Indira Gandhi Medical College and Research Institute, Pondicherry, India

${ }^{2}$ Department of Microbiology, Mahatma Gandhi Medical College and Research Institute, Pillaiyarkuppam, Pondicherry, India

${ }^{3}$ Department of Pediatrics, Sri Lakshmi Narayana Institute of Medical Sciences (SLIMS), Osudu, Agaram Village, Pondicherry, India

${ }^{4}$ Dept of Obstetrics and Gynaecology, Jawaharlal Institute of Postgraduate Medical Education and Research (JIPMER), Pondicherry, India

\begin{abstract}
Introduction: During the last few decades, group B Streptococcus (GBS) has emerged as an important pathogen. The major reservoirs for GBS are the vagina and the peri-anal regions/rectum, and the colonization of these regions is a risk factor for subsequent infection in pregnant women and newborns.

Methodology: A prospective study was performed to determine the prevalence of GBS colonization in the vagina and rectum of pregnant women and the antibiotic susceptibility pattern of the isolates. We also aimed to identify risk factors associated with GBS colonization. The vaginal and rectal swabs were inoculated in Todd-Hewitt broth and later subcultured on blood agar for isolation of GBS.

Results: A total of 300 pregnant women were enrolled in the study. GBS strains were isolated from seven out of 300 patients, corresponding to a colonization rate of $2.3 \%$. Of the seven patients carrying GBS, isolates were cultured only from vaginal swabs in two cases ( $28.6 \%)$, only from rectal swabs in two cases $(28.6 \%)$ from both vaginal and rectal swabs in three cases $(42.9 \%)$. Heavy colonization was present only in $42.9 \%(3 / 7)$ of antenatal women. None of the seven isolates were resistant to penicillin or clindamycin, while one isolate (14.3\%) was resistant to erythromycin and five isolates $(71.4 \%)$ were resistant to tetracycline. Multigravid women and those with previous spontaneous abortion were more frequently colonized by GBS.

Conclusion: The GBS colonization rate in our study was low. No resistance to penicillin or clindamycin was seen, while the majority of the isolates were resistant to tetracycline.
\end{abstract}

Key words: Group B Streptococcus; colonization; prevalence; drug resistance; risk factors

J Infect Dev Ctries 2011; 5(8):592-595.

(Received 03 September 2010 - Accepted 22 January 2011)

Copyright (C) 2011 Sharmila et al. This is an open-access article distributed under the Creative Commons Attribution License, which permits unrestricted use, distribution, and reproduction in any medium, provided the original work is properly cited.

\section{Introduction}

Group B Streptococcus (GBS) has re-emerged as a major pathogen during the last few decades [1]. The vagina and the peri-anal regions/rectum are the major reservoirs for GBS, and the colonization of these regions is a risk factor for subsequent infection in pregnant women and newborns [2]. About $50-60 \%$ of infants born to colonized mothers have positive GBS cultures from skin and mucous membranes, and 1-2\% of these colonized newborns develop invasive GBS infection [3].

The rate of GBS colonization in the vagina and/or rectum among pregnant women varies with ethnic group, geographic area and age [4-6]. Maternal colonization by GBS was observed to range from $4 \%$ to $40 \%$ in several studies conducted worldwide [4-8]. Identification of maternal colonization by GBS during pregnancy is very important for taking preventive measures, such as antibiotic prophylaxis, against neonatal disease [9].

To reduce the incidence of neonatal disease caused by GBS, the Centers for Disease Control and Prevention recommends the use of intrapartum antibiotic prophylaxis in pregnant women who are vaginorectal carriers of GBS [10]. Penicillin is recommended as the first-line agent for intrapartum antibiotic prophylaxis, while ampicillin is considered as an acceptable alternative [10]. In penicillin-allergic 
Table 1. Association between risk factors and GBS colonization

\begin{tabular}{lllll}
\hline Risk factors & $\begin{array}{l}\text { Pregnant women } \\
\text { with GBS } \\
\text { colonization } \\
\text { (n=7) }\end{array}$ & $\begin{array}{l}\text { Pregnant women } \\
\text { without GBS } \\
\text { colonization } \\
\text { (n= 293) }\end{array}$ & $\begin{array}{l}\text { Odds ratio } \\
\text { (95\% Confidence } \\
\text { interval) }\end{array}$ & P value \\
\hline Maternal age $($ mean \pm SD) & $24.57 \pm 2.23$ & $23.25 \pm 2.96$ & - & 0.24 \\
Multigravida & $5(71.4 \%)$ & $100(34.1 \%)$ & $4.82(0.91$ to 25.32$)$ & 0.05 \\
Previous spontaneous abortion & $2(28.6 \%)$ & $17(5.8 \%)$ & $6.49(1.17$ to 35.97) & 0.07 \\
\hline
\end{tabular}

women, who are not at high risk for anaphylaxis, cefazolin is considered the agent of choice for intrapartum chemoprophylaxis because of its narrow spectrum of activity and ability to achieve high intraamniotic concentrations [10]. In pregnant women at high risk for penicillin anaphylaxis, clindamycin or erythromycin is recommended [10]. Although GBS strains are generally susceptible to penicillin with only occasional resistance, they show varying resistance to erythromycin, clindamycin and tetracycline [11-13]. Therefore, a regional knowledge of the resistance profile of GBS will be useful for administration of appropriate antibiotics for prophylaxis. The present study was undertaken to determine the prevalence of GBS colonization in the vaginas and rectums of pregnant women and the antibiotic susceptibility pattern of the isolates. We also aimed to identify risk factors associated with GBS colonization.

\section{Methodology}

A prospective observational cohort study was conducted between September 2006 and June 2008, at Jawaharlal Institute of Postgraduate Medical Education and Research (JIPMER), a tertiary care teaching institute located at Puducherry, South India. Pregnant women with a gestational age of 35-37 weeks, attending the antenatal out-patient department of JIPMER, were enrolled in the study and followed up until delivery. A detailed obstetric history was taken especially to identify the risk factors for GBS colonization. Those with history of intake of antibiotics during the past two weeks and/or preexisting medical disorders complicating pregnancy were excluded from the study. This study was approved by the Research and Ethical committees of Jawaharlal Institute of Postgraduate Medical Education and Research (JIPMER) and informed consent was obtained from the patients.
Two vaginal swabs and two rectal swabs were collected from each pregnant women included in the study. One vaginal swab and one rectal swab were directly plated on blood agar, while the second vaginal and rectal rectal swabs were placed in ToddHewitt broth, an enrichment medium for GBS, and later subcultured on blood agar. Repeat swabs were taken during subsequent antenatal visits of the patient to our hospital. GBS strains were identified based on the colony morphology, beta hemolysis and characteristic biochemical reactions [14], and was confirmed by latex agglutination test (Strepto Plus, Biomerieux Marcy l'Etoile, France). Heavy colonization was defined as culture of GBS from direct plating rather than only from selective broth [10]. Kirby-Bauer disc diffusion method was performed according to the Clinical Laboratory Standards Institute (CLSI) guidelines, to determine the antibiotic susceptibility patterns of the isolates [15]. Inducible clindamycin resistance was detected by disk diffusion using the D-zone test [11]. Streptococcus pneumoniae ATCC 49619 was used for quality control of antibiotic susceptibility testing.

Data entry and analysis were done using statistical software SPSS for Windows Version 16.0 (SPSS Inc, Chicago, IL, USA). The Chi-square test and Fisher's exact test were used to compare the two groups. $P$ values $<0.05$ were considered statistically significant.

\section{Results}

A total of 300 pregnant women were enrolled in the study. The age of the participants ranged from 18 years to 39 year with a mean $\pm \mathrm{SD}$ of $23.28 \pm 2.95$ years. GBS strains were isolated from seven out of 300 patients, corresponding to a colonization rate of $2.33 \%$. Of the seven colonised patients, two had strains cultured only from vaginal swabs, while two had strains isolated only from rectal swabs and 
Table 2. GBS colonization rates reported in different studies

\begin{tabular}{|c|c|c|c|c|}
\hline Reference no & Place & Year of isolation & Sample size & Colonization rate \\
\hline Elbaradie et al $^{5}{ }^{5}$ & Egypt & $2006-2007$ & 95 & $17.9 \%$ \\
\hline Orrett $^{17}$ & West Indies & 2003 & 201 & $32.9 \%$ \\
\hline Nwachukwu et al. ${ }^{2}$ & Nigeria & $2002-2003$ & 200 & $9.0 \%$ \\
\hline Motlova et al. ${ }^{12}$ & Czech Republic & $2001-2002$ & 586 & $29.3 \%$ \\
\hline Kulkarni et al. ${ }^{1}$ & India & 1998-1999 & 317 & $2.52 \%$ \\
\hline Regan et al. ${ }^{4}$ & USA & 1984-1989 & 13,646 & $21 \%$ \\
\hline
\end{tabular}

another three had strains isolated simultaneously from both the vaginal and rectal swabs. The isolation rate of GBS using direct plating on blood agar was $3 / 300(1 \%)$, while the isolation rate following selective enrichment with Todd-Hewitt broth was $7 / 300$ (2.3\%; $P$ value 0.3387 ). Accordingly, heavy colonization was present only in $3 / 7(42.9 \%)$ of antenatal women. One isolate from each patient was used for further study; of these, none were resistant to penicillin or clindamycin, while one isolate was resistant to erythromycin and five isolates were resistant to tetracycline. The one isolate resistant to erythromycin was negative for inducible clindamycin resistance, but was resistant to tetracycline. Antibiotic prophylaxis was given to all seven women with GBS colonization. Repeat cultures, which were obtained after two weeks of treatment, were negative for GBS. None of the babies born to the colonized mothers suffered from any neonatal complications.

The association between risk factors and colonization of the maternal recto-vaginal region with GBS is summarized in Table 1 and indicates that multigravida is a risk factor for GBS colonization.

\section{Discussion}

In the last few decades GBS has gained importance due to its ability to cause serious neonatal infections. In developed countries GBS is a leading cause of sepsis and meningitis in neonates with a high case fatality rate of about $40 \%-80 \%$, yet the magnitude of this infection in developing countries such as India has not been adequately studied $[16,17]$.

In the present study, several isolates were obtained simultaneously from both the vaginal and rectal swabs, and some were isolated only from either rectal swabs or vaginal swabs. In a similar study, swabbing both the vagina and rectum was found to increase the yield substantially compared with sampling either the vagina or the rectum alone [1].

In the present study, the GBS colonization rate was $2.33 \%$ and $42.9 \%$ of the colonized women had heavy colonization. The GBS colonization rate in our study was relatively low compared to other reports (Table 2). However, our rate was similar to that of another Indian study reporting $2.52 \%$ colonization among pregnant women [1]. The Centers for Disease Control (CDC) has recommended use of selective enrichment broth to maximize the isolation of GBS, because when direct agar plating is used instead of selective enrichment broth, approximately $50 \%$ of women who are GBS carriers can be missed [10]. However, in the present study, despite using selective enrichment broth, we observed GBS colonization only in a small number of pregnant women (2.3\%), suggesting that maternal colonization with GBS is low in India.

The CDC recommends that it is important to monitor for the emergence of penicillin resistance among GBS, as it is one of the key sentinel events which may signal a need for revising the current guidelines [10]. All our GBS isolates were sensitive to penicillin. In studies conducted in different patient populations GBS was uniformly sensitive to penicillin $[1,2,18,19]$. The CDC advocates testing of GBS isolates for susceptibility to clindamycin and erythromycin, as they are the drugs of choice for penicillin-allergic women at high risk for anaphylaxis [10]. Although one of our isolates was found resistant to erythromycin, all were sensitive to clindamycin. In two different studies, $8 \%$ and $22 \%$ resistance to erythromycin were observed, while in other studies no resistance to erythromycin was observed, suggesting that GBS show variable resistance to erythromycin $[1,2,18,20]$.

In the present study, we observed that multigravid women were more often associated with 
GBS colonization, though it was not statistically significant. In a study from the West Indies, colonization rates were found to be significantly greater among multigravid women than primigravid women [18]. However, in another study involving Turkish pregnant women, parity was observed to be unrelated to GBS carriage [19]. Therefore, further studies are needed to confirm the correlation between parity and colonization by GBS. We observed that occurrence of previous spontaneous abortion may be associated with GBS colonization, but we could not prove a statistically significant association due to the small sample size.

To conclude, the GBS colonization rate was $2.33 \%$, of which $42.9 \%$ was associated with heavy colonization. The isolation rate of GBS strains was improved by selective enrichment. None of the isolates were resistant to penicillin or clindamycin, while $14.3 \%$ were resistant to erythromycin and $71.4 \%$ were resistant to tetracycline. Multigravida and occurrence of previous spontaneous abortion were observed to be possible risk factors for GBS colonization.

\section{References}

1. Kulkarni AA, Pawar SG, Dharmadhikari CA, Kulkarni RD (2001) Colonization of pregnant women and their newborn infants with group-B streptococci. Indian J Med Microbiol 19: 1-4.

2. Nwachukwu NC, Utsalo SJ, Kanu I, Anyanwu EC (2007) Genital Colonization of Group B Streptococcus at term pregnancy in Calabar, Nigeria. The Internet Journal of Pediatrics and Neonatology 7: 9.

3. Shet A and Ferrieri P (2004) Neonatal \& maternal group B streptococcal infections: A comprehensive review. Indian J Med Res 120: 141-150.

4. Allardice JG, Baskett TF, Seshia MM, Bowman N, Malazdrewicz R (1982) Perinatal group B streptococcal colonization and infection. Am J Obstet Gynecol 142: 617620.

5. Regan JA, Klebanoff MA, Nugent RP (1991) The epidemiology of group B streptococcal colonization in pregnancy. Vaginal Infections and Prematurity Study Group. Obstet Gynecol 77: 604-610.

6. Elbaradie SM, Mahmoud M, Farid M (2009) Maternal and neonatal screening for Group B streptococci by SCP B gene based PCR: a preliminary study. Indian J Med Microbiol 27: 17-21.

7. Hickman ME, Rench MA, Ferrieri P, Baker CJ (1999) Changing epidemiology of group $\mathrm{B}$ streptococcal colonization. Pediatrics 104: 203-209.

8. Moyo SR, Mudzori J, Tswana SA, Maeland JA (2000) Prevalence, capsular type distribution, anthropometric and obstetric factors of group B Streptococcus (Streptococcus agalactiae) colonization in pregnancy. Cent Afr J Med 46: 115-120.

9. Baker CJ and Barrett FF (1973) Transmission of group B streptococci among parturient women and their neonates. J Pediatr 83: 919-925.

10. Schrag S, Gorwitz R, Fultz-Butts K, Schuchat A (2002) Prevention of perinatal group B streptococcal disease. Revised guidelines from CDC. MMWR Recomm Rep 51: 122.

11. Clinical Laboratory Standards Institute (2007) Performance standards for antimicrobial susceptibility testing. Sixteenth informational supplement. CLSI document M100 - S 16. CLSI: Wayne, PA.

12. Andrews JI, Diekema DJ, Hunter SK, Rhomberg PR, Pfaller MA, Jones RN, Doern GV (2000) Group B streptococci causing neonatal bloodstream infection: antimicrobial susceptibility and serotyping results from SENTRY centers in the Western Hemisphere. Am J Obstet Gynecol 183: 859-862.

13. Motlova J, Strakova L, Urbaskova P, Sak P, Sever T (2004) Vaginal \& rectal carriage of Streptococcus agalactiae in the Czech Republic: incidence, serotypes distribution \& susceptibility to antibiotics. Indian J Med Res 119: 84-87.

14. Collee JG, Marmion BP, Fraser AG, Simmons A (1996) Mackie and McCartney's Practical medical microbiology. 14th edition. New York: Churchill Livingstone 978p.

15. Clinical Laboratory Standards Institute (2006) Performance standards for antimicrobial disk susceptibility tests. Approved standard, $9^{\text {th }}$ ed. CLSI document M2-A9. CLSI: Wayne, PA.

16. Prakash K, Ravindran PC, Sharma KB (1976) Group B beta haemolytic streptococci and their serological types in human infections. Indian J Med Res 64: 1001-1007.

17. Kishore K, Deorari AK, Paul VK, Singh M, Bhujwala RA (1986) Group B Streptococcus colonization \& neonatal outcome in north India. Indian J Med Res 84: 492-494.

18. Orrett FA (2003) Colonization with Group B streptococci in pregnancy and outcome of infected neonates in Trinidad. Pediatr Int 45: 319-323.

19. Eren A, Kucukercan M, Oguzoglu N, Unal N, Karateke A (2005) The carriage of group B streptococci in Turkish pregnant women and its transmission rate in newborns and serotype distribution. Turk J Pediatr 47: 28-33.

20. Dzowela T, Komolafe OO, Igbigbi A (2005) Prevalence of group B Streptococcus colonization in antenatal women at the Queen Elizabeth Central Hospital, Blantyre - a preliminary study. Malawi Medical Journal 17: 97-99.

\section{Corresponding author}

Dr. Vijayan. Sharmila

Assistant Professor

Department of Obstetrics \& Gynaecology

Indira Gandhi Medical College and Research Institute

Pondicherry, India

Telephone: 914132200479

Email: sharu_jipmer@yahoo.co.in

Conflict of interests: No conflict of interests is declared. 